\title{
LA=UR- 0900010
}

- Approved for public release; distribution is unlimited.

Title:

Intense lon Beams as a Tool for Opacity Measurements in Warm Dense Matter

Author(s): $\quad$ Joseph Abdallah, Jr., T-1

An Tauschwiz, J. Jacoby, J. A. Maruhn; University of Frankfurt, Germany

V. G. Novikov; Keldysh Inst. of Applied Math, Russia

A. Tauschwitz, E. Onkels, K. Witte; Darmstadt, Germany F. B. Rosmej, R. Schott; Ecole Polytechnique, France

Intended for:

Applied Physics B

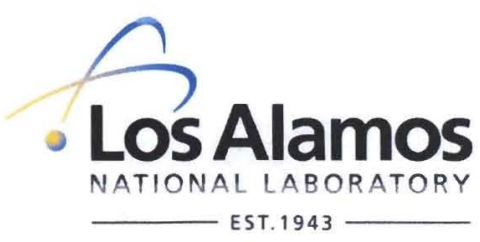

Los Alamos National Laboratory, an affirmative action/equal opportunity employer, is operated by the Los Alamos National Security, LLC for the National Nuclear Security Administration of the U.S. Department of Energy under contract DE-AC52-06NA25396. By acceptance of this article, the publisher recognizes that the U.S. Government retains a nonexclusive, royalty-free license to publish or reproduce the published form of this contribution, or to allow others to do so, for U.S. Government purposes. Los Alamos National Laboratory requests that the publisher identify this article as work performed under the auspices of the U.S. Department of Energy. Los Alamos National Laboratory strongly supports academic freedom and a researcher's right to publish; as an institution, however, the Laboratory does not endorse the viewpoint of a publication or guarantee its technical correctness. 


\title{
Intense ion beams as a tool for opacity measurements in warm dense matter
}

\author{
An. Tauschwitz ${ }^{1 \star}$, V.G. Novikov ${ }^{2}$, A. Tauschwitz ${ }^{3}$, F.B. Rosmej ${ }^{4,5}$, R. Schott ${ }^{5}$, J. Abdallah ${ }^{6}$, \\ E. Onkels ${ }^{3}$, J. Jacoby ${ }^{1}$, J.A. Maruhn ${ }^{1}$, K. Witte ${ }^{3}$ \\ 1 University of Frankfurt am Main, Germany \\ 2 Keldysh Institute of Applied Mathematics, Moscow, Russia \\ ${ }^{3}$ Gesellschaft für Schwerionenforschung, Darmstadt, Germany \\ ${ }^{4}$ Université Pierre et Marie Curie, Paris, France \\ ${ }^{5}$ Centre de Recherche LULI, Ecole Polytechnique, PAPD, Palaiseau, France \\ ${ }^{6}$ Los Alamos National Laboratory, USA
}

Received: date / Revised version: date

\begin{abstract}
Opacity measurements in warm dense matter (WDM) provide a valuable benchmark for the diverging theoretical models in this regime. Heating of thin foils with intense heavy ion beams allows to create isolated samples of warm dense matter suitable for experimental determination of frequency-dependent opacities. A prerequisite for the measurements is the isothermal expansion of the heated foil. Hydrodynamic simulations predict that this condition is fullfilled. The analysis shows that existing ion beam accelerators are capable to contribute to this field of research.
\end{abstract}

\section{Introduction}

Warm Dense Matter (WDM) refers to states with comparable thermal and Fermi energies and an ion-ion coupling parameter that exceeds unity. WDM can be considered as high-temperature condensed matter or as stronglycoupled plasma. In nature WDM occurs in the core of giant planets, in the laboratory it appears in every experiment in which plasma is created very quickly from a solid. Understanding of WDM is difficult because of the complexity of first principle theories and the lack of well defined experimental measurement [1].

The optical properties of strongly-coupled plasma are very sensitive to the electron energy spectrum defined by particle interactions. The opacity measurements of WDM will give a benchmark for theoretical approaches and clarify the influence of non-ideality on the absorption coefficients. In [2], Rosseland mean opacities obtained with Quantum Molecular Dynamics (QMD) simulations and the Los Alamos Light Element Detailed
Configuration OPacity code (LEDCOP) are compared. The calculations presented for warm dense $\mathrm{Al}$ disagree by up to several orders of magnitude. To the best of our knowledge, there are no experimental data available to validate the calculated results, which is due to the challenge of experimental measurements.

Intense charged particle beams provide a useful tool for creating isolated samples of warm dense matter. In this paper we want to address the measurement of frequency-dependent opacities in WDM created by an ion beam. The performed hydrodynamic calculations show that volumetric heating of thin foils by intense ion beams allows to create warm dense matter samples with a nearly constant temperature, which is essential for precise opacity measurements.

The target parameters for opacity measurements in ion beam produced WDM were chosen assuming an energy deposition of $10 \mathrm{~kJ} / \mathrm{g}$ in high-Z materials within a $100 \mathrm{~ns}$ ion pulse. These parameters are expected using bunches of $10^{10}$ uranium ions at the heavy ion synchrotron SIS-18 at the Gesellschaft für Schwerionenforschung, Darmstadt (GSI) in the year 2009. For first experiments lead can be chosen as material for opacity measurements because the highest temperatures are obtained by ion beam irradiation in high-Z materials. For a sub- $\mu \mathrm{m}$ foil target the plasma after the ion beam heating will have a density of about $\rho \approx 0.01 \mathrm{~g} / \mathrm{cm}^{3}$ and a temperature of $T \approx 2 \mathrm{eV}$. This corresponds for lead to a mean ion charge of $Z \sim 1-1.5$ according to the different models and a nonideality parameter $\Gamma \sim 0.4-0.6$.

In Sec. 2 the calculated radiation transmission in ion beam produced warm dense matter is presented together with hydrodynamic simulations. In Sec. 3 details of an experimental realization are discussed. Conclusions are given in Sec. 4.

\footnotetext{
* e-mail: an.tauschwitz@gsi.de
} 


\section{Target modeling}

Intense ion beam pulses are typically long on the time scale of the hydrodynamic target responce. Energy deposition of $10 \mathrm{~kJ} / \mathrm{g}$ over a $100 \mathrm{~ns}$ heating time causes a strong expansion of the initially sub- $\mu \mathrm{m}$ thick foil. This results in a plasma slab with about $0.01 \mathrm{~g} / \mathrm{cm}^{3}$ density and $2 \mathrm{eV}$ temperature. Figure 1 shows the calculated transmission for the $\mathrm{Pb}$ plasma of $0.02 \mathrm{~cm}$ thickness with the above parameters. The calculations were made using two completely different models - the Saha model with continuum lowering [3] and the quasizone model (QZM) [4], which includes electron-ion interaction in the mean spherical cell approximation.

In the quasizone model the spectrum of the high density plasma is described by using a so-called quasizone. This is an energy band which allows to treat bound states for inner electrons, free states for continuum and intermediate electron states with energy close to zero, including resonances, in the same manner. The quasizone appears due to the special boundary conditions for wave functions - quasi-periodical conditions in mean spherical approximation. In this case the electron wave function is presented as an expansion over spherical harmonics. There is one harmonic for every bound state, several for weakly bound states and an infinite number of terms for the free states. Using the quasizone model and local density approximation for electron exchange and correlation effects it is possible to obtain the energy spectrum, equation of state (EOS) and photon absorption coefficients in a wide range of plasma temperatures and densities. However, the above model uses an average atom approximation, and its accuracy may be insufficient at the considered low temperatures.

On the other hand, the Saha approximation does not include interaction between particles in the plasma and works for dilute plasmas only. The density limit for the Saha approach at low temperatures is under question. The results presented in Fig. 1 demonstrate a big difference between the two approaches. This discrepancy is caused by both different ionization states (Saha model gives mean ion charge $Z_{0}=0.96, \mathrm{QZM} Z_{0}=1.08$ ) and a merging of spectral lines into broad bands in QZM. The Saha approximation of ideal plasma at such conditions is problematic and models which include the effects of ion-electron interactions give results very different from the ideal-plasma approach and between each other. Measurements can clarify the question how the strong interaction affects the plasma properties.

Hydrodynamic calculations were performed to predict the spatial distribution of the plasma parameters after the ion beam heating. The foil thickness was optimized according to the calculated radiation transmission. For the simulations the hydrodynamic code CAVEAT was used together with the SESAME equation of state for $\mathrm{Pb} \#$ 3200. The calculated temperature and density profiles along the ion beam axis in the initially $0.3 \mu \mathrm{m}$

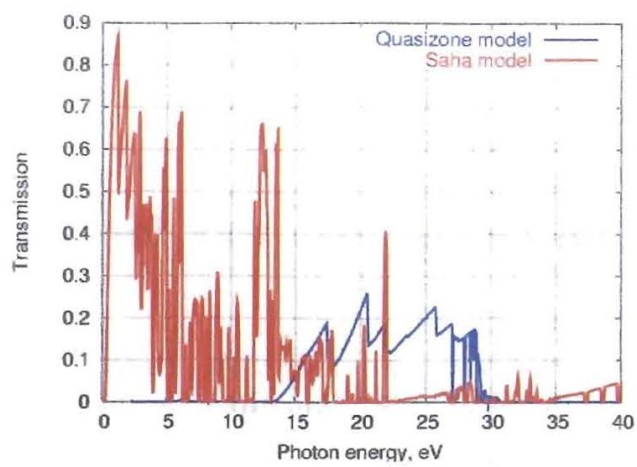

Fig. 1 Transmission of $\mathrm{Pb}$ plasma slab with $0.01 \mathrm{~g} / \mathrm{cm}^{3}$ density and $2 \mathrm{eV}$ temperature calculated with two different models; the plasma layer has a thickness of $0.02 \mathrm{~cm}$.
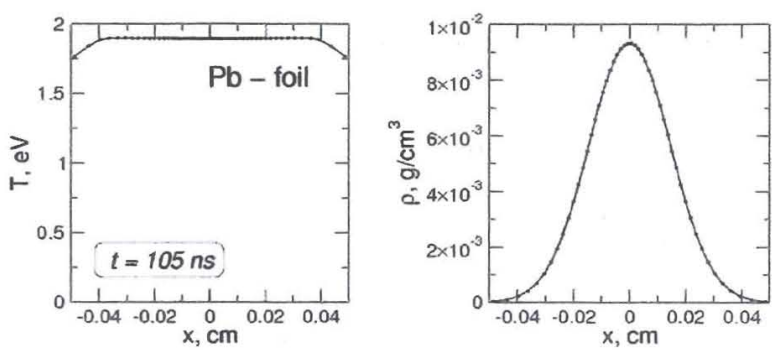

Fig. 2 Density and temperature distribution in an ion-beam heated lead foil at $\mathrm{t}=105 \mathrm{~ns}$.

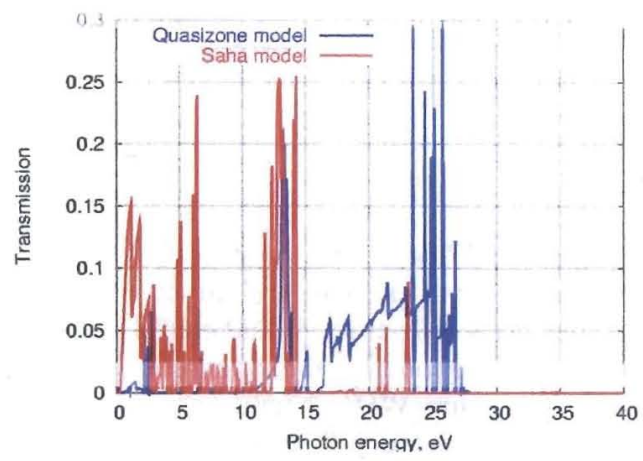

Fig. 3 Calculated transmission of the ion beam heated $\mathrm{Pb}$ plasma using two different models. The spatial distributions of density and temperature correspond to Fig. 2.

thick foil are presented in Fig. 2. The time $t=105 \mathrm{~ns}$ was chosen to ensure thermodynamic equilibration of the plasma after the energy deposition by the ion beam.

According to the simulation the temperature in the heated target of about $T=1.9 \mathrm{eV}$ is nearly constant, which is important for the interpretation of the measured plasma transmission. The density profile is gaussian with 
a maximum at the center with $\rho=0.009 \mathrm{~g} / \mathrm{cm}^{3}$. The density profile has to be measured and taken into account in the analysis of experimental transmission data. In Fig. 3 the plasma transmission calculated with the above density profile is presented. As expected, the density profile does not affect the transmitted spectrum significantly (compare to Fig. 1). Therefore, the plasma conditions achievable in ion beam heated foils are well suited for opacity measurements in the WDM - regime.

\section{Experimental realization}

For the described measurements it is not only important to create an isolated plasma sample with properties suitable for the opacity measurement, but also to have a backlighter source which covers the wavelength region of interest. Such source should be sufficiently intense and have a spectrum without pronounced line structure. A suitable backlighter can be created with the help of a high power laser, either by direct target heating or via a radiative hohlraum. This approach will be exploited at the future FAIR facility where a suitable laser will be available together with the intense ion beam.

For near term experiments an alternative solution can be to drive the backlighter with the same ion beam as the target foil for the opacity measurement. Such a source can be realized using an ion beam heated high$\mathrm{Z}$ foil placed in front of the target to be investigated, as shown in Fig. 4. Preferably the temperature of this backlighter foil is several times higher than the temperature of the target. For small temperature differences the accuracy of the measurement is reduced, since the selfemission of the target foil has to be subtracted from the recorded spectrum. Experimentally, the difference in the deposited energy between the backlighter and the target foils can be achieved by the displacement of the target foil from the waist of the ion beam focus. Calculations show that a heated gold foil of $1 \mu \mathrm{m}$ thickness emits radiation close to a black body spectrum with a temperature of about $2.3 \mathrm{eV}$ when heated with a beam of $15 \mathrm{~kJ} / \mathrm{g}$ energy deposition. The temperatures of both, the backlighter and the target foil can be determined by fitting the self emission to a Planckian curve. The calculated emission of the backlighter foil at $t=105 \mathrm{~ns}$ is presented at Fig. 5 in comparison with black body radiation of the same temperature.

The light that is transmitted through the target foil has to be spectrally resolved and recorded. A VUVspectrometer that allows a temporal resolution of few ns is required to perform the measurement. To determine the characteristics of the backlighter its spectrum has to be recorded separately. Likewise the emission spectrum of the target foil alone has to be measured. The brightness of an ion beam heated backlighter source is more than sufficient even if a large distance between the target foil and the spectrometer and short gating times

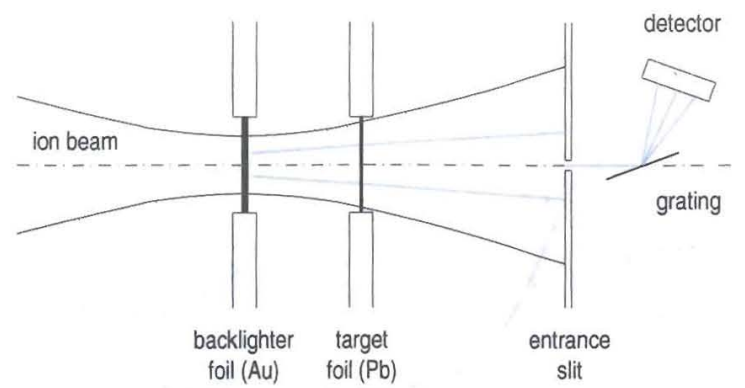

Fig. 4 Schematic view of the target-backlighter configuration.

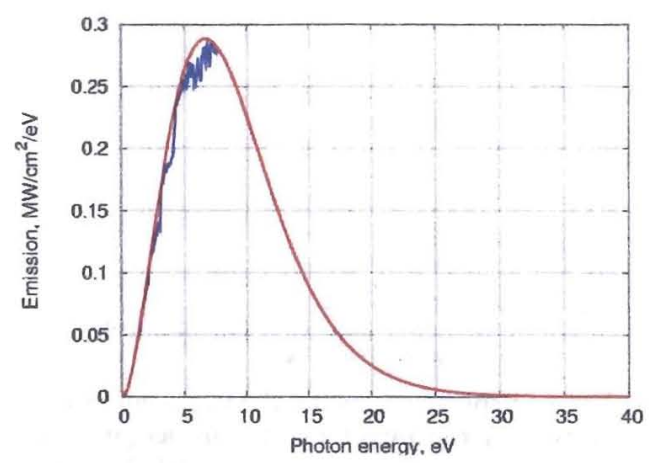

Fig. 5 Emission spectrum of the $1 \mathrm{~m} \mathrm{Au}$ foil heated to a temperature of $\mathrm{T} \simeq 2.3 \mathrm{eV}$ (blue line) and Planckian spectrum corresponding to the same temperature (red line).

are assumed. Nevertheless the signal to noise ratio is of concern. Neutrons originating in the foils are expected to be the main source for the background signal.

Figure 6 shows the calculated spectrum that will be observed behind the target foil. It includes the emission spectrum of the target foil and the light of the backlighter foil transmitted through the plasma target. The emission spectrum of the target has to be subtracted from the measured spectrum. The resulting spectrum has than to be divided by the emission spectrum of the backlighter to obtain the transmission. The calculated spectrum obtained with the help of the quasizone model (blue) is closer to a black body spectrum than the spectrum based on the Saha approach (red).

\section{Conclusions}

Intense ion beams are a promising tool for opacity measurements in warm dense matter. The parameters of the plasma samples after the ion beam irradiation are well suited for experiments. The isothermal expansion of the irradiated foils allows the precise interpretation of the measured data. A second foil heated with the same ion 

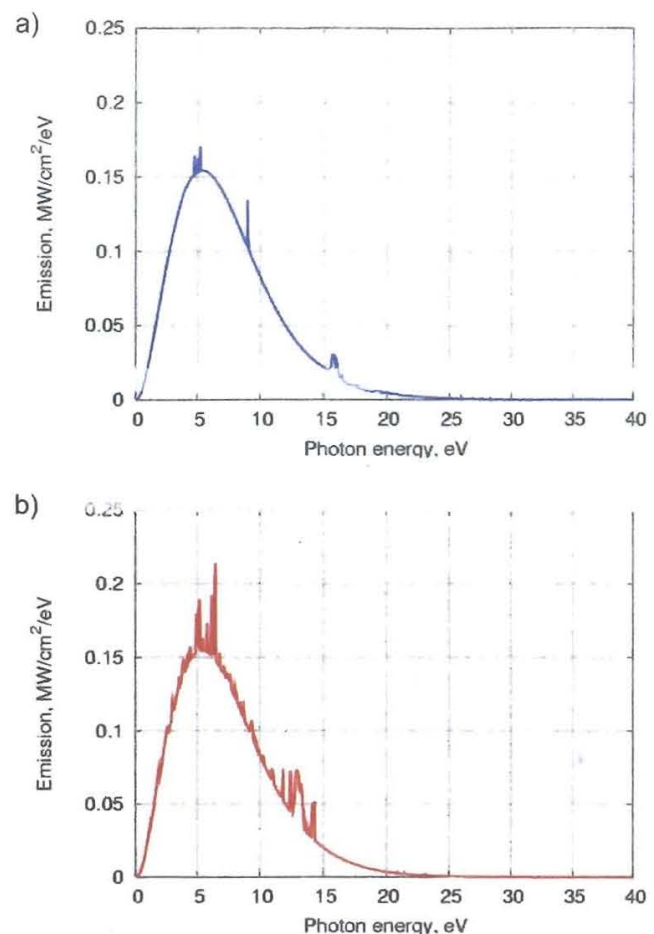

Fig. 6 The calculated spectrum behind the target foil. Density and temperature of the target plasma are as given in Fig. 2. a) calculation using the quasizone model, b) Saha approach.

beam can serve as a backlighter source. At present accelerator facilities like SIS-18 at GSI these opacity measurements can be performed.

Opacity measurements will be an essential part in the work of the WDM collaboration at FAIR [5]. Making use of the higher beam intensities, shorter ion bunches and the availability of a high power laser at the future facility will overcome many restrictions imposed by current machines. Plasmas of higher temperatures and densities can be created without the present limitation to high-Z materials. The high power laser will enable backlighter sources with a wide range of temperatures.

\section{Acknowledgments}

The authors would like to thank Mikhail Basko (ITEP, Moscow) and Klaus Eidmann (MPQ, Garching) for helpful discussions.

\section{References}

1. "Frontiers for discovery in high energy density physics", prepared by National task force on High Energy Density physics, July 20, 2004. Available at www.ofes.fusion.doe.gov/News/HEDPReport.pdf
2. S. Mazevet, M.P. Desjarlais, L.A. Collins et al., Phys. Rev. E 71, 016409 (2005)

3. D. Salzmann, Atomic physics in hot plasmas (New York Oxford, Oxford University Press, 1998)

4. A.F. Nikiforov, V.G. Novikov, V.B. Uvarov, Quantumstatistical models of hot dense matter: methods for computation opacity and equation of state (Birkhauser Verlag, Basel-Boston-Berlin, 2005)

5. "WDM - radiative properties of warm dense matter produced by intense heavy ion beams", FAIR Baseline Technical Report, vol. 5, 2006 (paragraph 5.3). Available at http://www.gsi.de/fair/reports/btr.html 